\title{
Expression of vasohibin-1 and -2 predicts poor prognosis among patients with squamous cell carcinoma of the esophagus
}

\author{
YAMATO NINOMIYA $^{1}$, SOJI OZAWA ${ }^{1}$, JUNYA OGUMA ${ }^{1}$, AKIHITO KAZUNO ${ }^{1}$, \\ MIHO NITTA $^{1}$, HIROSHI KAJIWARA ${ }^{2}$ and YASUFUMI SATO ${ }^{3}$ \\ Departments of ${ }^{1}$ Gastroenterological Surgery and ${ }^{2}$ Pathology, Tokai University School of Medicine, Isehara, \\ Kanagawa 259-1193; ${ }^{3}$ Department of Vascular Biology, Institute of Development, \\ Aging and Cancer, Tohoku University, Sendai, Miyagi 980-9575, Japan
}

Received December 29, 2017; Accepted June 28, 2018

DOI: $10.3892 / \mathrm{ol} .2018 .9249$

\begin{abstract}
Vasohibin (VASH) -1 and -2 are novel angiogenic regulators. The aim of the present study was to assess the prognostic values of VASH1 expression and VASH2 expression in esophageal squamous cell carcinoma (ESCC). A total of 209 patients with ESCC were investigated. Resected tumor specimens were immunostained using anti-CD34 antibody, anti-VASH1 antibody and anti-VASH2 antibody. The ratio of the microvessels density and the VASH1 density as the VASH1-positive ratio were defined and the patients were divided into two groups (a high VASH1 group and a low VASH1 group) according to the average value. The patients were also divided into two groups (a high VASH2 group and a low VASH2 group) according to VASH2 expression upon immunostaining. The clinical outcomes of these two groups were then evaluated. The high VASH1 group contained 106 patients (50.7\%). The high VASH2 group contained 48 patients (23.0\%). Long-term survival was significantly poorer in the high VASH1 group compared with that in the low VASH1 group. A slight correlation between VASH1 expression and VASH2 expression was observed. The low VASH1/low VASH2 group had a better prognosis than the other three groups with different combinations of VASH1 and VASH2 expression levels. The present study showed that high VASH1 expression and high VASH2 expression may be novel independent predictors of a poor prognosis in patients with ESCC and that a slight correlation between VASH1 and VASH2
\end{abstract}

Correspondence to: Professor Soji Ozawa, Department of Gastroenterological Surgery, Tokai University School of Medicine, 143 Shimokasuya, Isehara, Kanagawa 259-1193, Japan

E-mail: sozawa@tokai.ac.jp

Abbreviations: VASH1, vasohibin-1; VASH2, vasohibin-2; ESCC, esophageal squamous cell carcinoma; VEGF, vascular endothelial growth factor; FGF-2, fibroblast growth factor 2; MVD, microvessels density; OS, overall survival; DFS, disease-free survival; HCC, hepatocellular carcinoma

Key words: angiogenesis, esophageal cancer, prognostic factor, VASH1, VASH2 expression existed. The present findings suggest that combined evaluation of VASH1 and VASH2 expression should provide an improved understanding of their clinicopathological features.

\section{Introduction}

According to the Comprehensive Registry of Esophageal Cancer in Japan 2009, the 5-year survival rate of esophageal cancer is $53.1 \%$ (1). Compared with other gastrointestinal cancers, the malignant potential of esophageal cancer is thought to be relatively high, while the prognosis is relatively poor (2).

Tumor microenvironmental factors, such as tumor angiogenesis, are critical for enabling tumor growth, progression and distant metastasis (3). Angiogenesis is well defined as the formation of neovessels and is a key event in tumor oncogenesis. Therefore, tumor angiogenesis is considered a useful index of malignancy. We previously reported an association between angiogenesis and the malignancy of esophageal cancer $(4,5)$. Here, we focus on vasohibins (VASH), novel angiogenesis regulators.

Components of the VASH family have been recently identified as novel negative feedback regulators of angiogenesis (6,7). The VASH family consists of two subtypes, VASH1 and its homologue VASH2; these subtypes appear to have distinct roles in angiogenesis regulation (8). VASH1 is mainly expressed in vascular endothelial cells located behind the sprouting front of new blood vessel and acts to terminate angiogenesis, which is induced by angiogenesis stimulators such as vascular endothelial growth factor (VEGF) and fibroblast growth factor 2 (FGF-2) $(6,9)$. On the other hand, VASH2 is mainly expressed on cancer cells and CD11b-positive mononuclear cells infiltrating germinating regions where angiogenesis is active and has the effect of promoting angiogenesis at sprouting front (6). In several types of cancer, VASH1 has been reported as a prognostic factor correlated with cancer angiogenesis and malignancy (10-17). However, its involvement in squamous cell carcinoma of esophagus has not been studied. VASH2 has been reported as a prognostic factor in patients with pancreatic cancer (18), but its involvement in squamous cell carcinoma or esophageal cancer has not been studied. In addition, the simultaneous 
expression of both VASH1 and VASH2 in one disease and the correlations between the resulting expression patterns and prognosis have also not been investigated.

In this study, we examined the expressions of VASH1 and VASH2 in esophageal squamous cell carcinoma (ESCC) and investigated the relationship between these expression patterns and patient prognosis. We also examined whether the expressions of VASH1 and VASH2 can be used as biomarkers for the malignancy of esophageal cancer.

\section{Patients and methods}

Patients. We investigated 209 patients with thoracic ESCC who underwent curative surgical resection between January 2006 and December 2009 at Tokai University Hospital (Kanagawa, Japan). A transthoracic esophagectomy and three-field lymphadenectomy were regarded as the standard surgical treatment during this period. Node-positive patients received adjuvant chemotherapy with cisplatin and 5-fluorouracil. We excluded patients who had received neoadjuvant chemotherapy or neoadjuvant chemoradiotherapy and whose pathological examination results were pT1a or pT4. Patients were followed up using endoscopy, computed tomography, ultrasonography, and blood tests including tumor marker levels every 6 months for 5 years after surgery. The esophageal cancers were classified according to the Japanese classification of esophageal cancer, 11th edition $(19,20)$. The study was approved by Institutional Review Board for Clinical Research, Tokai University.

Immunohistochemistry. The specimens had been fixed in $10 \%$ formalin and were embedded in paraffin. The paraffin blocks were cut into $5-\mu \mathrm{m}$ thick sections. After deparaffinization and hydration, autoclaving was performed for $30 \mathrm{~min}$ at $120^{\circ} \mathrm{C}$ in Target Retrieval Solution, pH 6.0 (Dako, Glostrup, Denmark). To block endogenous peroxidase activity, the specimens were left at room temperature for $10 \mathrm{~min}$ in $3 \%$ hydrogen peroxidase/methanol. The specimens were then incubated for $10 \mathrm{~min}$ at room temperature in $5 \%$ goat serum (Nichirei Bioscience, Tokyo, Japan). Anti-VASH1 antibody (6) and anti-VASH2 antibody (21) diluted at 1:500 and anti-CD34 antibody (Nichirei Biosciences) diluted at 1:100 were used as the primary antibodies, and the specimens were reacted with these antibodies overnight at $4^{\circ} \mathrm{C}$. After washing with PBS, the specimens were reacted with Histofine Simple Stain MAX PO (M) (Nichirei Biosciences) as a secondary antibody for $30 \mathrm{~min}$ at room temperature. The reacted specimens were visualized using 3,3'-diaminobenzamine tetrahydrochloride and $0.005 \%$ hydrogen peroxide in $50 \mathrm{mmol} / 1$ Tris- $\mathrm{HCl}(\mathrm{pH} 7.5)$ and were counterstained with hematoxylin to detect nuclei. The appropriate negative control slides for CD34 antigen, VASH1, and VASH2 were prepared by substituting the primary antibody with PBS. H\&E staining was also performed on all sections (Fig. 1A).

Evaluation of immunostaining. The tumor microvessels were visualized using CD34 immunohistochemistry. The area with the highest number of vessels (hot spot) (22) was scanned at a low magnification $(\mathrm{x} 40)$ and at least 5 hot spots were evaluated; the hot spot with the highest number of vessels was then selected, and the vessels were counted at a high magnification (x200; $\left.0.74 \mathrm{~mm}^{2}\right)$ (23). VASH1-positive vessels were counted for the hot spot with the highest number of vessels positive for CD34. The number of each vessel type was then converted into a number per one square millimeter. We regarded the number of microvessels in the hot spot as the microvessels density (MVD) and the number of VASH1-positive vessels in the same spot as the VASH1 density (Fig. 1B and C). We defined the ratio of the MVD and VASH1 density as the VASH1-positive ratio and divided the patients into two groups (a high VASH1 group and a low VASH1 group) according to the average value $(11,13)$. We also divided the patients into two groups (a high MVD group and a low MVD group) according to the average MVD value. The clinical outcomes of the groups were then evaluated.

VASH2-positive tumor cells showed positive staining for VASH2 in the tumor cell cytoplasm (Fig. 1D). The extent of VASH2 immunostaining of a VASH2-positive tumor was scored as follows, according to the percentage of the positively stained area of the tumor relative to the total tumor area: $0(0 \%), 1(1-50 \%)$, and 2 (51-100\%) (18). Patients with a score of 2 were classified into the high VASH2 group, and those with a score of 0 or 1 were classified into the low VASH2 group. The clinical outcomes of these two groups were then evaluated. Furthermore, to examine the relationship between VASH1 expression and VASH2 expression, we divided the patients into 4 groups (a high VASH1/high VASH2 group, a high VASH1/low VASH2 group, a low VASH1/high VASH2 group, and a low VASH1/low VASH2 group) according to a combination of the high or low VASH1 status and the high or low VASH2 status. We then examined how VASH1/VASH2 expression was correlated with prognosis.

Statistical analysis. The associations among each clinicopathological factor, the recurrence pattern, VASH1 and VASH2 expression, and the MVD of the tumor were analyzed using a $\mathrm{x}^{2}$ test or a Student's t-test. The survival times were measured from the day of surgery, and death from esophageal cancer was regarded as the outcome. The overall survival curve and disease-free survival curve were drawn using the Kaplan-Meier method, and differences were assessed using the log-rank test. The Cox proportional hazards regression model was used for the univariate and multivariate analyses. $\mathrm{P}<0.05$ was considered to indicate a statistically significant difference. The analyses were performed using SPSS statistics (version 23.0; IBM Japan, Tokyo, Japan).

\section{Results}

The average VASH1-positive ratio was $0.467 \pm 0.255$; the high VASH1 group contained 106 patients $(50.7 \%)$, and the low VASH1 group contained 103 patients (49.3\%). Significant differences in pT $(\mathrm{P}=0.028)$, lymph node metastasis number $(\mathrm{P}=0.005)$, pTNM stage $(\mathrm{P}=0.012)$, and adjuvant chemotherapy $(\mathrm{P}=0.011)$ were observed between the two groups (Table I).

The high VASH2 group contained 48 patients (23.0\%), and the low VASH2 group contained 161 patients (77.0\%). No significant differences in clinical parameters were observed between the two groups (Table II).

The average MVD was 88.2; the high MVD group contained 95 patients $(45.5 \%)$, and the low MVD group contained 
A

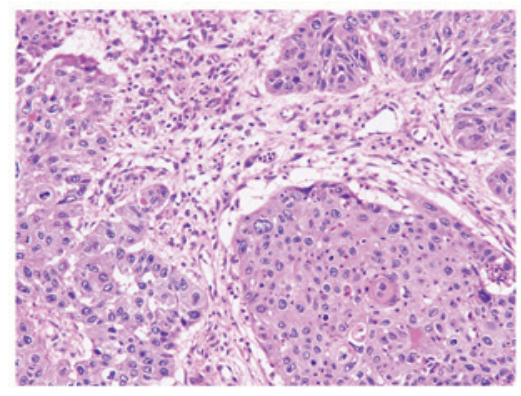

$\mathrm{C}$

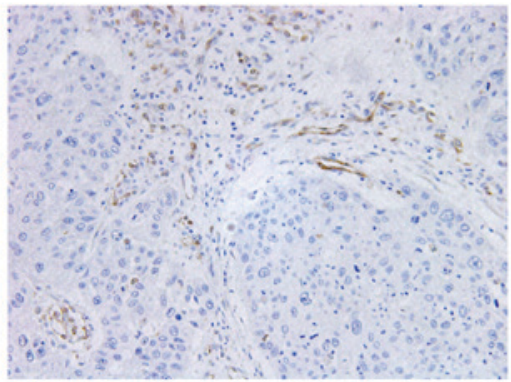

B

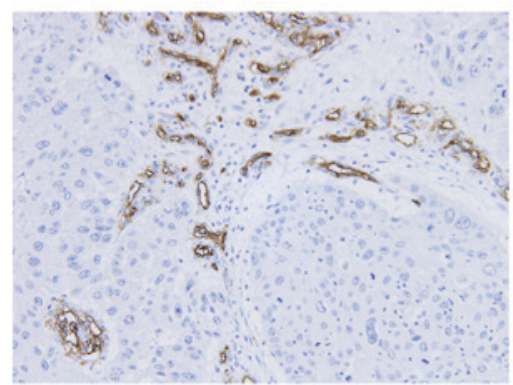

D

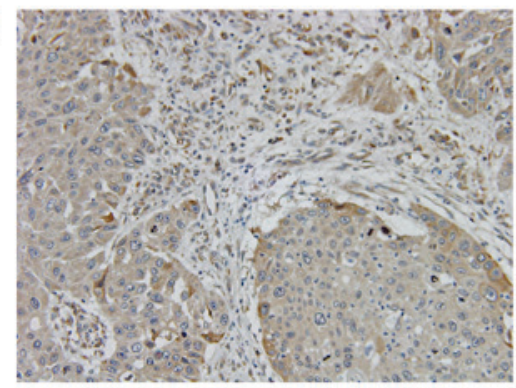

Figure 1. (A) H\&E staining and immunostaining for (B) CD34, (C) VASH1, (D) VASH2. Magnification, x200 for all panels. VASH, vasohibin.

114 patients (54.5\%). However, no significant differences in clinical parameters were observed between the two groups.

In the overall survival (OS) analysis, univariate analyses revealed that differentiation $(\mathrm{P}<0.001)$, lymphatic invasion $(\mathrm{P}=0.002)$, venous invasion $(\mathrm{P}<0.001), \mathrm{pT}(\mathrm{P}<0.001), \mathrm{pN}$ $(\mathrm{P}<0.001)$, VASH1 expression level $(\mathrm{P}<0.001)$, and VASH2 expression level $(\mathrm{P}<0.001)$ were prognostic factors. No relation between MVD and patient outcome was seen. A multivariate analysis also showed that venous invasion $(\mathrm{P}=0.005), \mathrm{pN}$ $(\mathrm{P}=0.004)$, VASH1 expression level $(\mathrm{P}=0.040)$, and VASH2 expression level $(\mathrm{P}=0.003)$ were prognostic factors (Table III). Survival rate was significantly poorer in the high VASH1 group than in the low VASH1 group (Fig. 2A), and in the high VASH2 group than in the low VASH2 group (Fig. 2B). No significant differences were seen between the high MVD and the low MVD groups. In terms of disease-free survival (DFS), a univariate analysis revealed that differentiation $(\mathrm{P}=0.002)$, lymphatic invasion $(\mathrm{P}=0.016)$, venous invasion $(\mathrm{P}<0.001)$, $\mathrm{pT}$ $(\mathrm{P}<0.001), \mathrm{pN}(\mathrm{P}<0.001)$, VASH1 expression level $(\mathrm{P}<0.001)$, VASH2 expression level $(\mathrm{P}=0.028)$, and adjuvant chemotherapy $(\mathrm{P}=0.003)$ were significant factors. As with the OS results, no correlation was seen between MVD and DFS. A multivariate analysis also showed that venous invasion $(\mathrm{P}=0.008)$, $\mathrm{pN}(\mathrm{P}<0.001)$, and VASH1 expression level $(\mathrm{P}=0.007)$ were significant factors (Table IV). DFS was significantly poorer in the high VASH1 group than in the low VASH1 group (Fig. 2C) and in the high VASH2 group than in the low VASH2 group (Fig. 2D). No significant differences were observed between the high MVD and the low MVD groups.

There was a positive correlation between VASH1 expression level and MVD (correlation coefficient 0.542, $\mathrm{P}<0.001$ ), but there was no correlation between VASH2 expression level and MVD.

We divided patients into 4 groups (a high VASH1/high VASH2 group, a high VASH1/low VASH2 group, a low VASH1/high VASH2 group, and a low VASH1/low VASH2 group) according to the combination of the high or low VASH1 status and the high or low VASH2 status. A slight correlation between VASH1 expression and VASH2 expression was seen $(\mathrm{P}=0.063)$ (Table V). The OS and the DFS were better in the low VASH1 and low VASH2 group than in the high VASH1 and/or high VASH2 group ( $\mathrm{P}<0.001)$ (Fig. $2 \mathrm{E}$ and $\mathrm{F})$.

Regarding the recurrence patterns, we examined lymph node metastasis, organ metastasis and both types of metastasis in terms of VASH1 and VASH2 expression. No significant differences were observed between each of the two groups.

\section{Discussion}

In the present study, we performed immunostaining using resected specimens of ESCC and examined the relationship between VASH1 and VASH2 expression and clinical oncological factors. A high VASH1 expression level and high VASH2 expression level were shown to be prognostic factors for OS. These results were presumed to reflect the influence of VASH1 and VASH2 expression on tumor angiogenesis and tumor progression in ESCC. When patients were classified into 4 groups according to the combination of VASH1 and VASH2 expression patterns, the OS was better in the low VASH1 and low VASH2 group than in the high VASH1 and/or high VASH2 group. A slight relationship between VASH1 expression and VASH2 expression was also observed. The identification of an association between VASH1 and VASH2 expression patterns and patient outcome in the present study is likely to lead to the development of this biomarker as a new prognostic predictor of ESCC.

Angiogenesis is a phenomenon in which a new blood vessel network is formed from existing blood vessels (24). Tumor angiogenesis is thought to play an important role in tumor development and distant metastasis (3). In recent years, there have been several reports on the relationship between tumor angiogenesis and patient prognosis among patients with malignant tumors, leading to the development and clinical application of angiogenesis inhibitors (25-27). 
Table I. Comparison of clinicopathological factors between high VASH1 group and low VASH1 group.

\begin{tabular}{|c|c|c|c|c|}
\hline Variable & $\operatorname{Total}^{\mathrm{a}}(\%)$ & High VASH1 group $^{\mathrm{b}}(\%)$ & Low VASH1 $\operatorname{group}^{c}(\%)$ & P-value ${ }^{d}$ \\
\hline Age (years, mean) & 64.2 & 64.2 & 64.2 & 0.960 \\
\hline Sex & & & & 0.172 \\
\hline Male & $172(82)$ & $91(86)$ & $81(79)$ & \\
\hline Female & 37 (18) & $15(14)$ & $22(21)$ & \\
\hline Location of tumor & & & & 0.497 \\
\hline Upper & $21(10)$ & $8(7)$ & $13(13)$ & \\
\hline Middle & $118(56)$ & $59(56)$ & $59(57)$ & \\
\hline Lower & $70(34)$ & $39(37)$ & $31(30)$ & \\
\hline Size of tumor (mm, mean) & 49.4 & 50.0 & 49.2 & 0.894 \\
\hline Differentiation & & & & 0.170 \\
\hline Well & $65(31)$ & $33(31)$ & $32(31)$ & \\
\hline Moderate & $100(48)$ & $45(43)$ & $55(53)$ & \\
\hline Poorly & $44(21)$ & $28(26)$ & $16(16)$ & \\
\hline Lymphatic invasion & & & & 0.198 \\
\hline$(-)$ & $45(22)$ & $19(18)$ & $26(25)$ & \\
\hline$(+)$ & $164(78)$ & $87(82)$ & $77(75)$ & \\
\hline Venous invasion & & & & 0.075 \\
\hline$(-)$ & $65(31)$ & $27(25)$ & $38(37)$ & \\
\hline$(+)$ & $144(69)$ & $79(75)$ & $65(63)$ & \\
\hline INF & & & & 0.864 \\
\hline $\mathrm{a}$ & $17(8)$ & $9(8)$ & $8(8)$ & \\
\hline $\mathrm{b}$ & $162(78)$ & $80(76)$ & $82(80)$ & \\
\hline $\mathrm{c}$ & $30(14)$ & $17(16)$ & $13(12)$ & \\
\hline pT & & & & 0.028 \\
\hline $\mathrm{T} 1$ & $85(41)$ & $33(31)$ & $52(51)$ & \\
\hline $\mathrm{T} 2$ & $37(18)$ & $21(20)$ & $16(15)$ & \\
\hline $\mathrm{T} 3$ & $87(41)$ & $52(49)$ & $35(34)$ & \\
\hline $\mathrm{pN}$ & & & & 0.056 \\
\hline No & $70(33)$ & $27(25)$ & $43(42)$ & \\
\hline $\mathrm{N} 1$ & $63(30)$ & $36(34)$ & $27(26)$ & \\
\hline $\mathrm{N} 2$ & $62(30)$ & $31(29)$ & $31(30)$ & \\
\hline N3 & $9(4)$ & $7(7)$ & $2(2)$ & \\
\hline N4 & $5(3)$ & $5(5)$ & $0(0)$ & \\
\hline Lymph node metastasis number & 2.7 & 3.6 & 1.8 & 0.005 \\
\hline pTNM stage & & & & 0.012 \\
\hline I & $46(22)$ & $18(17)$ & $28(27)$ & \\
\hline II & $76(36)$ & $32(30)$ & $44(43)$ & \\
\hline III & $82(39)$ & $51(48)$ & $31(30)$ & \\
\hline $\mathrm{IV}^{\mathrm{a}}$ & $5(3)$ & $5(5)$ & $0(0)$ & \\
\hline Adjuvant chemotherapy & & & 0.011 & \\
\hline$(-)$ & $95(45)$ & $39(37)$ & $56(54)$ & \\
\hline$(+)$ & $114(55)$ & $67(63)$ & $47(46)$ & \\
\hline
\end{tabular}

${ }^{\mathrm{a}} \mathrm{n}=209,{ }^{\mathrm{b}} \mathrm{n}=106,{ }^{\mathrm{c}} \mathrm{n}=103$, ${ }^{\mathrm{d}}$ age and size of tumor were analyzed using a t-test, and the other factors were analysed with a $x^{2}$-test. VASH1, vasohibin-1; INF, infiltrative growth.

VASH1 is involved in the suppression of angiogenesis and is thought to be expressed not only during physiological angiogenesis, but also during pathological angiogenesis in vascular endothelial cells at the site of angiogenesis (28). To date, the expression of VASH1 and its effect on patient prognosis has been reported for breast cancer (10), hepatocellular carcinoma 
Table II. Comparison of clinicopathological factors between high VASH2 group and low VASH2 group.

\begin{tabular}{|c|c|c|c|c|}
\hline Variable & $\operatorname{Total}^{\mathrm{a}}(\%)$ & High VASH2 group $^{\mathrm{b}}(\%)$ & Low VASH2 group $^{c}(\%)$ & P-value ${ }^{d}$ \\
\hline Age (years, mean) & 64.2 & 62.4 & 64.7 & 0.402 \\
\hline Sex & & & & 0.517 \\
\hline Male & $164(78)$ & $30(63)$ & $134(83)$ & \\
\hline Female & $45(22)$ & $18(37)$ & $27(17)$ & \\
\hline Location of tumor & & & & 0.458 \\
\hline Upper & $21(10)$ & $2(5)$ & $19(12)$ & \\
\hline Middle & $118(56)$ & $29(60)$ & $89(56)$ & \\
\hline Lower & $70(34)$ & $17(35)$ & $53(32)$ & \\
\hline Size of tumor (mm, mean) & 49.4 & 53.8 & 48.1 & 0.513 \\
\hline Differentiation & & & & 0.087 \\
\hline Well & $65(31)$ & $14(29)$ & $51(32)$ & \\
\hline Moderate & $100(48)$ & $18(38)$ & $82(51)$ & \\
\hline Poorly & $44(21)$ & $16(33)$ & $28(17)$ & \\
\hline Lymphatic invasion & & & & 0.893 \\
\hline$(-)$ & $45(22)$ & $10(21)$ & $35(22)$ & \\
\hline$(+)$ & $164(78)$ & $38(79)$ & $126(78)$ & \\
\hline Venous invasion & & & & 0.298 \\
\hline$(-)$ & $65(31)$ & $12(25)$ & $53(33)$ & \\
\hline$(+)$ & $144(69)$ & $36(75)$ & $108(67)$ & \\
\hline INF & & & & 0.510 \\
\hline $\mathrm{a}$ & $14(7)$ & $1(2)$ & $13(8)$ & \\
\hline $\mathrm{b}$ & $167(80)$ & $41(85)$ & $126(78)$ & \\
\hline $\mathrm{c}$ & $28(13)$ & $6(13)$ & $22(14)$ & \\
\hline pT & & & & 0.161 \\
\hline $\mathrm{T} 1$ & $85(40)$ & $14(29)$ & $71(44)$ & \\
\hline $\mathrm{T} 2$ & $27(13)$ & $8(17)$ & $29(18)$ & \\
\hline $\mathrm{T} 3$ & $87(47)$ & $26(54)$ & $61(38)$ & \\
\hline $\mathrm{pN}$ & & & & 0.110 \\
\hline N0 & $70(33)$ & $11(23)$ & $59(36)$ & \\
\hline $\mathrm{N} 1$ & $63(30)$ & $11(23)$ & $52(33)$ & \\
\hline $\mathrm{N} 2$ & $62(30)$ & $22(46)$ & $40(25)$ & \\
\hline N3 & $9(4)$ & $2(4)$ & $7(4)$ & \\
\hline N4 & $5(3)$ & $2(4)$ & $3(2)$ & \\
\hline Lymph node metastasis number & 2.7 & 3.3 & 2.6 & 0.355 \\
\hline pTNM stage & & & & 0.198 \\
\hline I & $46(22)$ & $6(13)$ & $40(25)$ & \\
\hline II & $76(36)$ & $15(31)$ & $61(38)$ & \\
\hline III & $81(39)$ & $25(52)$ & $57(35)$ & \\
\hline $\mathrm{IV}^{\mathrm{a}}$ & $6(3)$ & $2(4)$ & $3(2)$ & \\
\hline Adjuvant chemotherapy & & & & 0.696 \\
\hline$(-)$ & $95(45)$ & $23(49)$ & $72(45)$ & \\
\hline$(+)$ & $114(55)$ & $25(51)$ & $89(55)$ & \\
\hline
\end{tabular}

${ }^{a} n=209,{ }^{b} n=48,{ }^{c} n=161,{ }^{d}$ age and size of tumor were analyzed using a t-test, and the other factors were analysed with a $x^{2}$-test. VASH2, vasohibin-2; INF, infiltrative growth.

(HCC) (11), urothelial carcinoma (12), prostate cancer (13), colorectal carcinoma (14), non-small cell lung cancer (15), renal cell carcinoma (16) and head and neck squamous cell carcinoma (17). In each of these cancers, the prognosis is poor for the high VASH1 expression group. In terms of squamous cell carcinoma, Yoshinaga et al Hosaka et al and Torii et al 
Table III. Univariate and multivariate survival analysis of overall survival for ESCC patients by Cox's proportional hazard model.

\begin{tabular}{|c|c|c|c|c|c|c|}
\hline \multirow[b]{2}{*}{ Variable } & \multicolumn{3}{|c|}{ Univariate analysis } & \multicolumn{3}{|c|}{ Multivariate analysis } \\
\hline & HR & $95 \% \mathrm{CI}$ & P-value & HR & $95 \% \mathrm{CI}$ & P-value \\
\hline \multicolumn{7}{|l|}{ Age (years) } \\
\hline$<65$ vs. $\geq 65$ & 1.005 & $0.661-1.527$ & 0.932 & & & \\
\hline \multicolumn{7}{|l|}{ Sex } \\
\hline Male vs. female & 1.053 & $0.604-1.837$ & 0.855 & & & \\
\hline \multicolumn{7}{|l|}{ Locaton of tumor } \\
\hline Upper vs. middle and lower & 0.833 & $0.418-1.662$ & 0.604 & & & \\
\hline \multicolumn{7}{|l|}{ Size of tumor $(\mathrm{mm})$} \\
\hline$<49$ vs. $\geq 49$ & 1.521 & $0.961-2.406$ & 0.074 & & & \\
\hline \multicolumn{7}{|l|}{ Differentiation } \\
\hline Well and moderate vs. poorly & 2.357 & $1.515-3.667$ & $<0.001$ & & & \\
\hline $\begin{array}{l}\text { Lymphatic invasion } \\
(-) \text { vs. (+) }\end{array}$ & 3.374 & $1.558-7.307$ & 0.002 & & & \\
\hline \multicolumn{7}{|l|}{ Venous invasion } \\
\hline$(-)$ vs. $(+)$ & 4.077 & $2.165-7.676$ & $<0.001$ & 2.620 & $1.346-5.099$ & 0.005 \\
\hline $\begin{array}{l}\text { INF } \\
a \text { and } b \text { vs. c }\end{array}$ & 1.300 & $0.734-2.304$ & 0.368 & & & \\
\hline $\begin{array}{l}\text { pT } \\
1 \text { vs. } 2 \text { and } 3\end{array}$ & 2.960 & $1.796-4.878$ & $<0.001$ & & & \\
\hline \multicolumn{7}{|l|}{$\mathrm{pN}$} \\
\hline$(-)$ vs. (+) & 3.956 & $2.190-7.144$ & $<0.001$ & 2.494 & $1.350-4.610$ & 0.004 \\
\hline \multicolumn{7}{|l|}{ MVD } \\
\hline Low vs. high & 0.711 & $0.502-1.185$ & 0.236 & & & \\
\hline \multicolumn{7}{|l|}{ VASH1 expression } \\
\hline Low vs. high & 2.291 & $1.478-3.552$ & $<0.001$ & 1.604 & $1.021-2.519$ & 0.040 \\
\hline \multicolumn{7}{|l|}{ VASH2 expression } \\
\hline Low vs. high & 2.370 & $1.521-3.691$ & $<0.001$ & 1.996 & $1.268-3.142$ & 0.003 \\
\hline $\begin{array}{l}\text { Adjuvant chemotherapy } \\
(-) \text { vs. }(+)\end{array}$ & 1.507 & $0.976-2.328$ & 0.064 & & & \\
\hline
\end{tabular}

ESCC, esophageal squamous cell carcinoma; INF, infiltrative growth; MVD, microvessels density; HR, hazard ratio; CI, confidence interval; VASH, vasohibin.

previously reported the effects on cervical carcinoma (22), lung cancer (29) and head and neck squamous cell carcinoma (17), but these reports included both squamous cell carcinoma and adenocarcinoma and the numbers of cases were not large. The present study targeted only patients with squamous cell carcinoma and included a relatively large number of cases; both of these features are considered to be valuable from the viewpoint of examining the expression of VASH1 in squamous cell carcinoma.

In the present study, we used the VASH1-positive ratio as a means of evaluating VASH1 expression. VASH1 is expressed on the vascular endothelium at the site of angiogenesis, and CD34 is expressed not only at the site of angiogenesis, but also in existing vascular endothelium (30). Because of this feature, we can calculate the proportion of new blood vessels to all vessels by determining the ratio of both vessel types; this ratio is thought to reflect the activity of angiogenesis.
Since VASH1 is an angiogenesis inhibiting factor, a high VASH1 expression level was expected to suppress angiogenesis, resulting in a better prognosis. However, the present results showed that a high VASH1 expression level was correlated with a poorer outcome. One interpretation of this correlation is that VASH1 is induced by angiogenic factors, such as VEGF and FGF-2 $(6,9)$. Indeed, the intensity of this VASH1 positively correlated with that of VEGF in cancer cells $(10,13)$. However, VASH1 is degraded and inactivated after its secretion in the tumor microenvironment (31). Thus, the immunohistochemical staining of VASH1 in endothelial cells may not specify its anti-angiogenic activity, but simply reflects the response of endothelial cells to angiogenic stimulation.

Based on the above interpretation, the activity of angiogenesis in the high VASH1 group was thought to be higher than that in the low VASH1 group. As a result, tumor 
A

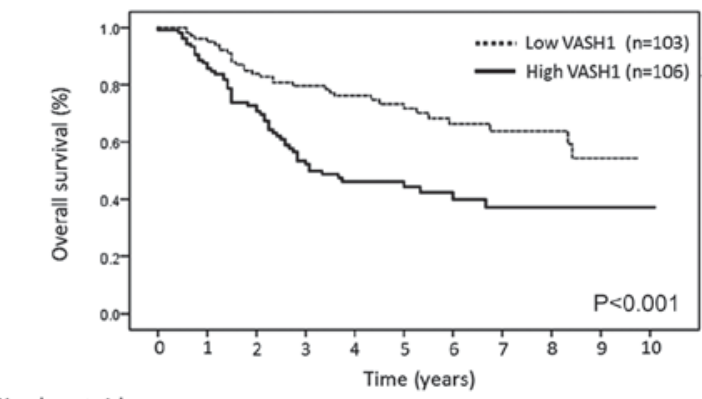

Number at risk

$\begin{array}{llllllllllll}\text { Low VASH1 } & 103 & 98 & 87 & 83 & 80 & 77 & 74 & 73 & 73 & 71 & 71\end{array}$

$\begin{array}{llllllllllll}\text { High VASH1 } & 106 & 91 & 76 & 59 & 54 & 53 & 51 & 50 & 50 & 50 & 50\end{array}$

\section{C}

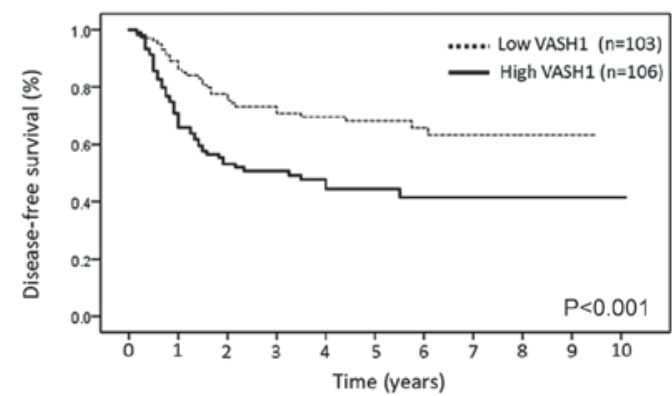

Number at risk

$\begin{array}{llllllllllll}\text { Low VASH1 } & 103 & 89 & 79 & 75 & 74 & 73 & 72 & 71 & 71 & 71 & 71\end{array}$

$\begin{array}{llllllllllll}\text { High VASH1 } & 106 & 71 & 59 & 57 & 53 & 53 & 52 & 52 & 52 & 52 & 52\end{array}$

E

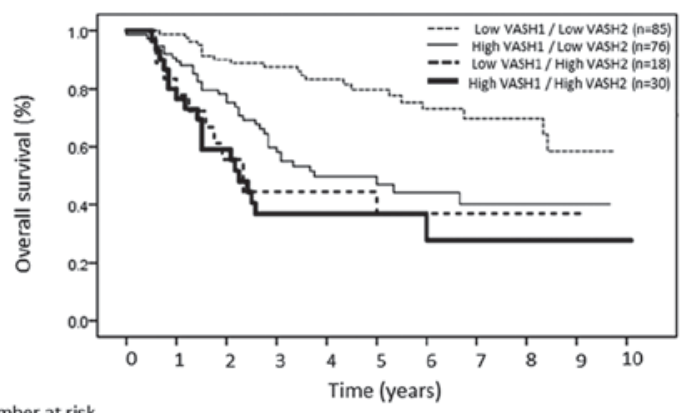

Number at risk

$\begin{array}{llllllrrrrrr}\text { Low VASH1/LOW VASH2 } & 85 & 84 & 76 & 75 & 72 & 70 & 67 & 66 & 66 & 64 & 64 \\ \text { High VASH1/Low VASH2 } & 76 & 68 & 58 & 47 & 42 & 41 & 40 & 39 & 39 & 39 & 39 \\ \text { Low VASH1/High VASH2 } & 18 & 14 & 10 & 8 & 8 & 7 & 7 & 7 & 7 & 7 & 7 \\ \text { High VASH1/High VASH2 } & 30 & 23 & 18 & 18 & 18 & 18 & 17 & 17 & 17 & 17 & 17\end{array}$

B

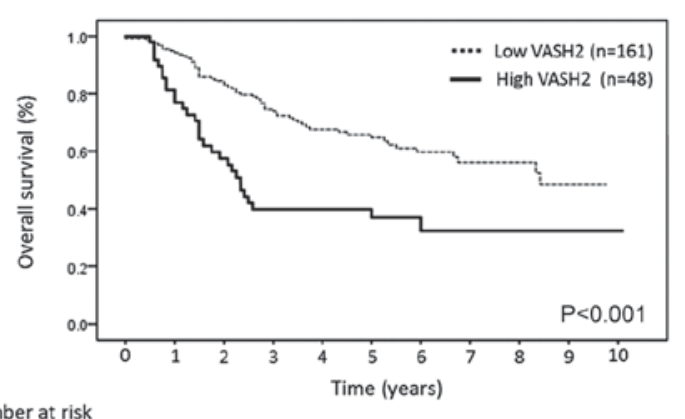

Number at risk

$\begin{array}{llllllllllll}\text { Low VASH2 } & 161 & 152 & 135 & 122 & 114 & 111 & 107 & 105 & 105 & 103 & 103\end{array}$ $\begin{array}{llllllllllll}\text { High VASH2 } & 48 & 37 & 28 & 20 & 20 & 19 & 18 & 18 & 18 & 18 & 18\end{array}$

D

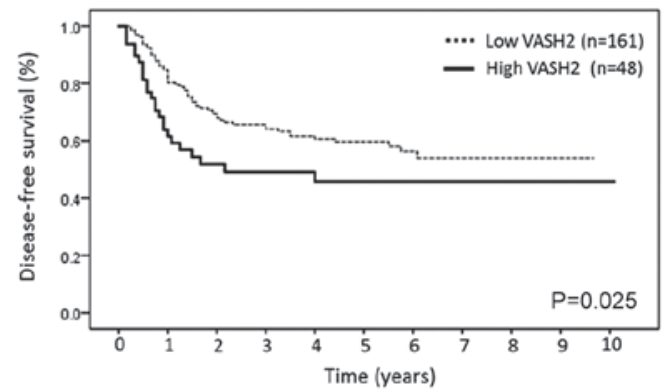

Number at risk

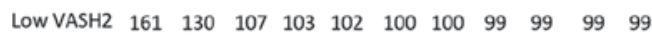

$\begin{array}{llllllllllll}\text { High VASH2 } & 48 & 30 & 22 & 21 & 20 & 20 & 20 & 20 & 20 & 20 & 20\end{array}$

\section{$\mathbf{F}$}

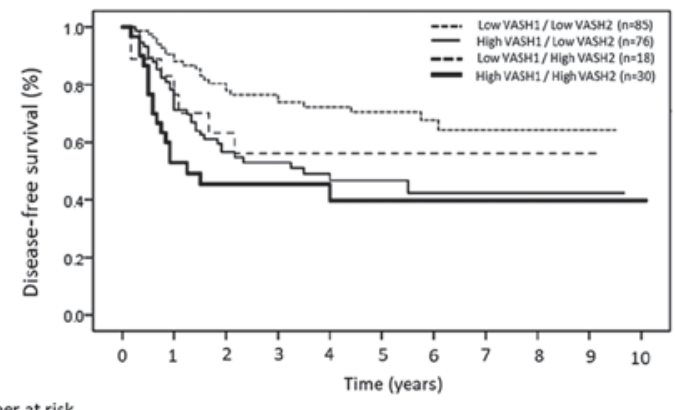

Number at risk

$\begin{array}{llllllllllll}\text { LOW VASH1/LOW VASH2 } & 85 & 75 & 67 & 64 & 63 & 62 & 61 & 60 & 60 & 60 & 60\end{array}$ $\begin{array}{llllllllllll}\text { High VASH1/Low VASH2 } & 76 & 55 & 45 & 43 & 40 & 40 & 39 & 39 & 39 & 39 & 39\end{array}$

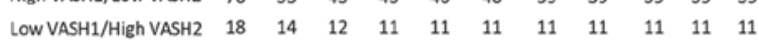
$\begin{array}{llllllllllll}\text { High VASH1/High VASH2 } & 30 & 16 & 14 & 14 & 13 & 13 & 13 & 13 & 13 & 13 & 13\end{array}$

Figure 2. Overall survival according to the (A) VASH1 and (B) VASH2 expression. Disease-free survival according to the (C) VASH1 and (D) VASH2 expression. Overall survival according to the (E) VASH1 and VASH2 expression. Disease-free survival according to the (F) VASH1 and VASH2 expression. VASH, vasohibin.

growth and metastasis were thought to have been increased, resulting in a poorer prognosis. In the present study, no difference in the recurrence pattern was seen between the two groups.

VASH2, a homologue of VASH1, is characterized as a pro-angiogenic factor (6). Indeed, the expression of VASH2 in human serous ovarian adenocarcinoma and HCC, and that promotes angiogenesis and accelerates tumor growth $(32,33)$. The relationship between the expression of VASH2 and patient prognosis has been reported for pancreatic ductal adenocarcinoma (18). In this cancer type, the prognosis was relatively poor for the high VASH2 expression group. Similarly, in the present study, the outcomes of the VASH2-positive cases of ESCC were poor. Since, as with VASH1, no studies have examined the relation between VASH2 expression and prognosis for esophageal cancer and squamous cell carcinoma, the present study was considered to provide valuable information regarding VASH2.

As VASH2 has an angiogenesis promoting effect, the expression of VASH2 may correlate with tumor angiogenesis. However, there was no correlation between VASH 2 expression level and MVD. Besides, several reports have described a new function of VASH2. In one study, an autocrine and paracrine mode of action for VASH2 was found to enhance the expression of FGF-2 and VEGF via the upregulation of nuclear factor- $\kappa \mathrm{B}$ in HCC cells (33). Furthermore, VASH2 
Table IV. Univariate and multivariate survival analysis of disease free survival for ESCC patients by Cox's proportional hazard model.

\begin{tabular}{|c|c|c|c|c|c|c|}
\hline \multirow[b]{2}{*}{ Variable } & \multicolumn{3}{|c|}{ Univariate analysis } & \multicolumn{3}{|c|}{ Multivariate analysis } \\
\hline & HR & $95 \% \mathrm{CI}$ & P-value & HR & $95 \%$ CI & P-value \\
\hline \multicolumn{7}{|l|}{ Age (years) } \\
\hline$<65$ vs. $\geq 65$ & 0.940 & $0.613-1.442$ & 0.778 & & & \\
\hline \multicolumn{7}{|l|}{ Sex } \\
\hline Male vs. female & 0.799 & $0.434-1.474$ & 0.473 & & & \\
\hline \multicolumn{7}{|l|}{ Locaton of tumor } \\
\hline Upper vs. middle and lower & 0.758 & $0.378-1.518$ & 0.434 & & & \\
\hline \multicolumn{7}{|l|}{ Size of tumor (mm) } \\
\hline$<49$ vs. $\geq 49$ & 1.504 & $0.937-2.416$ & 0.091 & & & \\
\hline \multicolumn{7}{|l|}{ Differentiation } \\
\hline Well and moderate vs. poorly & 2.116 & $1.331-3.366$ & 0.002 & & & \\
\hline \multicolumn{7}{|l|}{ Lymphatic invasion } \\
\hline \multicolumn{7}{|l|}{ Venous invasion } \\
\hline$(-)$ vs. (+) & 2.990 & $1.682-5.314$ & $<0.001$ & 2.211 & $1.233-3.967$ & 0.008 \\
\hline \multicolumn{7}{|l|}{ INF } \\
\hline$a$ and $b$ vs. $c$ & 1.379 & $0.776-2.451$ & 0.273 & & & \\
\hline \multicolumn{7}{|l|}{ pT } \\
\hline 1 vs. 2 and 3 & 2.501 & $1.536-4.072$ & $<0.001$ & & & \\
\hline \multicolumn{7}{|l|}{$\mathrm{pN}$} \\
\hline$(-)$ vs. $(+)$ & 5.224 & $2.694-10.129$ & $<0.001$ & 4.047 & $2.062-7.944$ & $<0.001$ \\
\hline \multicolumn{7}{|l|}{ MVD } \\
\hline Low vs. high & 0.906 & $0.588-1.396$ & 0.655 & & & \\
\hline \multicolumn{7}{|l|}{ VASH1 expression } \\
\hline Low vs. high & 2.293 & $1.463-3.592$ & $<0.001$ & 1.862 & $1.183-2.930$ & 0.007 \\
\hline VASH2 expression & & & & & & \\
\hline Low vs. high & 1.703 & $1.060-2.736$ & 0.028 & & & \\
\hline $\begin{array}{l}\text { Adjuvant chemotherapy } \\
(-) \text { vs. (+) }\end{array}$ & 2.025 & $1.274-3.218$ & 0.003 & & & \\
\hline
\end{tabular}

ESCC, esophageal squamous cell carcinoma; INF, infiltrative growth; MVD, microvessels density; HR, hazard ratio; CI, confidence interval; VASH, vasohibin.

Table V. Correlation between VASH1 expression and VASH2 expression.

\begin{tabular}{lccc}
\hline Variable & $\begin{array}{c}\text { High VASH1 } \\
(\mathrm{n}=106)\end{array}$ & $\begin{array}{c}\text { Low VASH1 } \\
(\mathrm{n}=103)\end{array}$ & P-value \\
\hline High VASH2 $(\mathrm{n}=48)$ & $30(14)$ & $18(9)$ & $\mathrm{P}=0.063$ \\
Low VASH2 $(\mathrm{n}=161)$ & $76(36)$ & $85(41)$ & \\
\hline
\end{tabular}

Parenthesis indicate \%. VASH, vasohibin.

has been found not only to accelerate angiogenesis, but also to promote HCC cell proliferation (33). In ovarian serous adenocarcinoma cells, the expression of VASH2 was inversely correlated with that of miR-200 (32). VASH2 promotes epithelial-to-mesenchymal transition of cancer cells by modulating TGF- $\beta$ signaling. $(34,35)$. Therefore, VASH 2 possesses other tumor-promoting functions, such as the induction of invasion and migration (36). These effects of VASH2 might also affect prognosis.

The most important part of our present study is the combined analysis of VASH1 and VASH2 expression level on patient prognosis. From the viewpoint of angiogenesis, the group in which both the VASH1 and the VASH2 expression levels were not elevated was thought to have a relatively low level of malignancy and a better prognosis. Among high VASH1 cases $(n=106)$ with increased tumor angiogenic potential, low VASH2 group contained 76 cases whereas high 
VASH2 group contained 30 cases. We speculate that other angiogenic factors than VASH2 might be involved in high VASH1/low VASH2 cases. Although it is not significant, a slight correlation was observed between VASH1 expression and VASH2 expression, with high VASH1 cases tending to be more common in the high VASH2 group (Table V). High VASH1/high VASH2 cases $(n=30)$ might reflect this combination, indicating that the expression of VASH1 in endothelial cells was promoted by VASH2 in cancer cells. Nine percent of cases $(n=18)$ showed poor prognosis with low VASH1/high VASH2. We assume that the effect of VASH2 on cancer progression might be rather independent on tumor angiogenesis in this subgroup. Since no studies have investigated the combined expressions of VASH1 and VASH2 in cancers, the present study provides a valuable information and detailed prognostic predictions.

Regarding MVD, no association with prognosis or disease-free survival was seen. In previous reports examining both VASH1 expression and the MVD in breast cancer and upper urinary tract urothelial carcinoma, the MVD was not clearly correlated with either the degree of progression or patient prognosis $(10,12)$. One of the reported reasons was that the MVD corresponds to the number of completed vessels and includes vessels without the potential for tumor neovascularization (12). The reports suggested that VASH1 expression might be a more sensitive prognostic factor than MVD (10,12). Similarly, the present study suggested that VASH1 expression might be a more useful prognostic factor than the MVD in patients with ESCC.

In conclusion, the present study showed that high VASH1 expression and high VASH2 expression were new independent predictors of a poor prognosis in patients with ESCC and that a slight correlation between VASH1 and VASH2 expression existed. The combined evaluation of VASH1 and VASH2 expression should provide a better understanding of their clinicopathological features.

\section{Acknowledgements}

The authors would like to thank Ms. Izu Inada (Department of Gastroenterological Surgery, Tokai University School of Medicine, Isehara, Japan) for their data analysis support.

\section{Funding}

The present study was supported by the Japan Society for the Promotion of Science (JSPS) KAKENHI (grant no. 17K10609).

\section{Availability of data and materials}

The analyzed datasets generated during the study are available from the corresponding authors on reasonable request.

\section{Authors' contributions}

YN, SO, and YS conceived and designed the study; YN, SO and $\mathrm{HK}$ were involved in data acquisition; YN, SO and JO were involved data analysis; YN, SO, JO, AK, MN and YS were involved in data interpretation. All authors have read and approval the final manuscript.

\section{Ethics approval and consent to participate}

The Institutional Review Board of Tokai University (Isehara, Japan) approved the study protocol and all patients provided written informed consent prior to obtaining the samples.

\section{Patient consent for publication}

Not applicable.

\section{Competing interests}

The authors declare that they have no competing interests.

\section{References}

1. Tachimori Y, Ozawa S, Numasaki H, Fujishiro M, Matsubara $\mathrm{H}$, Oyama T, Shinoda M, Toh Y, Udagawa $\mathrm{H}$ and Uno T; Registration Committee for Esophageal Cancer of the Japan Esophageal Society: Comprehensive registry of esophageal cancer in japan, 2009. Esophagus 13: 110-137, 2016.

2. Ito Y, Miyashiro I, Ito H, Hosono S, Chihara D, Nakata-Yamada K, Nakayama M, Matsuzaka M, Hattori M, Sugiyama $\mathrm{H}$, et al: Long-term survival and conditional survival of cancer patients in Japan using population-based cancer registry data. Cancer Sci 105: 1480-1486, 2014.

3. Ellis LM and Hicklin DJ: VEGF-targeted therapy: Mechanisms of anti-tumor activity. Nat Rev Cancer 8: 579-591, 2008.

4. Shih C, Ozawa S, Ando N, Ueda M and Kitajima M: Vascular endothelial growth factor expression predicts outcome and lymph node metastasis in squamous cell carcinoma of the esophagus. Clin Cancer Res 6: 1161-1168, 2000.

5. Takeuchi H, Ozawa S, Shih CH, Ando N, Kitagawa Y, Ueda M and Kitajima M: Loss of p16INK4a expression is associated with vascular endothelial growth factor expression in squamous cell carcinoma of the esophagus. Int J Cancer 109: 483-490, 2004.

6. Watanabe K, Hasegawa Y, Yamashita H, Shimizu K, Ding Y, Abe M, Ohta H, Imagawa K, Hojo K, Maki H, et al: Vasohibin as an endothelium-derived negative feedback regulator of angiogenesis. J Clin Invest 114: 898-907, 2004.

7. Sato Y: The vasohibin family: Novel regulators of angiogenesis. Vascul Pharmacol 56: 262-266, 2012.

8. Kimura H, Miyashita H, Suzuki Y, Kobayashi M, Watanabe K, Sonoda H, Ohta H, Fujiwara T, Shimosegawa T and Sato Y: Distinctive localization and opposed roles of vasohibin-1 and vasohibin-2 in the regulation of angiogenesis. Blood 113: 4810-4818, 2009.

9. Shimizu K, Watanabe K, Yamashita H, Abe M, Yoshimatsu H, Ohta $\mathrm{H}$, Sonoda $\mathrm{H}$ and Sato Y: Gene regulation of a novel angiogenesis inhibitor, vasohibin, in endothelial cells. Biochem Biophys Res Commun 327: 700-706, 2005.

10. Tamaki K, Moriya T, Sato Y, Ishida T, Maruo Y, Yoshinaga K, Ohuchi N and Sasano H: Vasohibin-1 in human breast carcinoma: A potential negative feedback regulator of angiogenesis. Cancer Sci 100: 88-94, 2009.

11. Murakami K, Kasajima A, Kawagishi N, Sekiguchi S, Fujishima F, Watanabe M, Sato Y, Ohuchi N and Sasano H: The prognostic significance of vasohibin 1-associated angiogenesis in patients with hepatocellular carcinoma. Hum Pathol 45: 589-597, 2014.

12. Miyazaki Y, Kosaka T, Mikami S, Kikuchi E, Tanaka N, Maeda T, Ishida M, Miyajima A, Nakagawa K, Okada Y, et al: The prognostic significance of vasohibin-1 expression in patients with upper urinary tract urothelial carcinoma. Clin Cancer Res 18: 4145-4153, 2012.

13. Kosaka T, Miyazaki Y, Miyajima A, Mikami S, Hayashi Y, Tanaka N, Nagata H, Kikuchi E, Nakagawa K, Okada Y, et al: The prognostic significance of vasohibin-1 expression in patients with prostate cancer. Br J Cancer 108: 2123-2129, 2013.

14. Kitajima T, Toiyama Y, Tanaka K, Saigusa S, Kobayashi M, Inoue Y, Mohri Y and Kusunoki M: Vasohibin-1 increases the malignant potential of colorectal cancer and is a biomarker of poor prognosis. Anticancer Res 34: 5321-5329, 2014. 
15. Zhang T, Yu TT, Zhang DM, Hou XM, Liu XJ, Zhao D and Shan L: Vasohibin-1 expression detected by immunohistochemistry correlates with prognosis in non-small cell lung cancer. Med Oncol 31: 963, 2014.

16. Mikami S, Oya M, Kosaka T, Mizuno R, Miyazaki Y, Sato Y and Okada Y: Increased vasohibin-1 expression is associated with metastasis and poor prognosis of renal cell carcinoma patients. Lab Invest 13: 854-862, 2017.

17. Torii C, Hida Y, Shindoh M, Akiyama K, Ohga N, Maishi N, Ohiro Y, Ono M, Totsuka Y, Kitagawa Y, et al: Vasohibin-1 as a novel prognostic factor for head and neck squamous cell carcinoma. Anticancer Res 37: 1219-1225, 2017.

18. Kim JC, Kim KT, Park JT, Kim HJ, Sato Y and Kim HS: Expression of vasohibin-2 in pancreatic ductal adenocarcinoma promotes tumor progression and is associated with a poor clinical outcome. Hepatogastroenterology 62: 251-256, 2015.

19. Japan Esophageal Society: Japanese classification of esophageal cancer, 11th edition: Part I. Esophagus 14: 1-36, 2017.

20. Japan Esophageal Society: Japanese classification of esophageal cancer, 11th edition: Part II and III. Esophagus 14: 37-65, 2017.

21. Koyanagi T, Suzuki Y, Komori K, Saga Y, Matsubara S, Fujiwara $\mathrm{H}$ and Sato $\mathrm{Y}$ : Targeting human vasohibin-2 by a neutralizing monoclonal antibody for anti-cancer treatment. Cancer Sci 108: 512-519, 2017.

22. Yoshinaga K, Ito K, Moriya T, Nagase S, Takano T, Niikura H, Sasano H, Yaegashi and Sato Y: Roles of intrinsic angiogenesis inhibitor, vasohibin, in cervical carcinomas. Cancer Sci 102: 446-451, 2011.

23. Weidner N, Semple JP, Welch WR and Folkman J: Tumor angiogenesis and metastasis-correlation in invasive breast carcinoma. N Engl J Med 324: 1-8, 1991.

24. Sato Y: Is vasohibin-1 more than angiogenesis inhibition? J Biochem 149: 229-230, 2011.

25. Roviello G, Petrioli R, Marano L, Polom K, Marrelli D, Perrella A and Roviello F: Angiogenesis inhibitors in gastric and gastroesophageal junction cancer. Gastric Cancer 19: 31-41, 2016.

26. Reck M, von Pawel J, Zatloukal P, Ramlau R, Gorbounova V, Hirsh V, Leighl N, Mezger J, Archer V, Moore N, et al: Overal survival with cisplatin-gemcitabine and bevacizumab or placebo as first-line therapy for nonsquamous non-small-cell lung cancer: Results from a randomised phase III trial (AVAiL). Ann Oncol 21: 1804-1809, 2010
27. Kabbinavar FF, Hambleton J, Mass RD, Hurwitz Hl, Bergsland E and Sarkar S: Combined analysis of efficacy: The addition of bevacizumab to fluorouracil/leucovorin improves survival for patients with metastatic colorectal cancer. J Clin Oncol 23: 3706-3712, 2005.

28. Nasu T, Maeshima Y, Kinomura M, Hirokoshi-Kawahara K, Tanabe K, Sugiyama H, Sonoda H, Sato Y and Makino H: Vasohibin-1, a negative feedback regulator of angiogenesis, ameliorates renal alterations in a mouse model of diabetic nephropathy. Diabetes 58: 2365-2375, 2009.

29. Hosaka T, Kimura H, Heishi T, Suzuki Y, Miyashita H, Ohta H, Sonoda H, Moriya T, Suzuki S, Kondo T and Sato Y: Vasohibin-1 expression in endothelium of tumor blood vessels regulates angiogenesis. Am J Pathol 175: 430-439, 2009.

30. Ho JW,PoonRT, Sun CK, Xue WC and Fan ST: Clinicopathological and prognostic implications of endoglin (CD105) expression in hepatocellular carcinoma and its adjacent non-tumorous liver. World J Gastroenterol 11: 176-181, 2005.

31. Saito M, Suzuki Y, Yano S, Miyazaki T and Sato Y: Proteolytic inactivation of anti-angiogenic vasohibin-1 by cancer cells. J Biochem 160: 227-232, 2016.

32. Takahashi Y, Koyanagi T, Suzuki Y, Saga Y, Kanomata N, Moriya T, Suzuki M and Sato Y: Vasohibin-2 expressed in human serous ovarian adenocarcinoma accelerates tumor growth by promoting angiogenesis. Mol Cancer Res 10: 1135-1146, 2012.

33. Xue X, Gao W, Sun B, Xu Y, Han B, Wang F, Zhang Y, Sun J, Wei J, Li Z, et al: Vasohibin 2 is transcriptionally activated and promotes angiogenesis in hepatocellular carcinoma. Oncogene 32: 1724-1734, 2013.

34. Tu M, Lu C, Lv N, Wei J, Lu Z, Xi C, Chen J, Guo F, Jiang K, Li Q, et al: Vasohibin 2 promotes human luminal breast cancer angiogenesis in a non-paracrine manner via transcriptional activation of fibroblast growth factor 2. Cancer Lett 383: 272-281, 2016.

35. Norita R, Suzuki Y, Furutani Y, Takahashi K, Yoshimatsu Y, Podyma-Inoue KA, Watabe T and Sato Y: Vasohibin-2 is required for epithelial-mesenchymal transition of ovarian cancer cells by modulating transforming growth factor- $\beta$ signaling. Cancer Sci 108: 419-426, 2017.

36. Koyanagi T, Saga Y, Takahashi Y, Suzuki Y, Suzuki M and Sato Y: Downregulation of vasohibin-2, a novel angiogenesis regulator, suppresses tumor growth by inhibiting angiogenesis in endometrial cancer cells. Oncol Lett 5: 1058-1062, 2013. 